\title{
A Novel Deep Learning Approach for Short Term Photovoltaic Power Forecasting Based on GRU-CNN Model
}

\author{
Mohammed Sabri ${ }^{1, *}$ and Mohammed El Hassouni ${ }^{2, * *}$ \\ ${ }^{1}$ LRIT, Mohammed V University in Rabat, Morocco \\ ${ }^{2}$ FLSH, Mohamed V University in Rabat, Morocco
}

\begin{abstract}
The integration of photovoltaic power brings the key to clean energy. However, the increasing proportion of photovoltaic (PV) energy in power systems due to the random and intermittent nature of solar energy resources is causing difficulties for system operators to dispatch PV power stations. To reduce the negative influence of the use of PV power, it is great significant to predict PV power accurately. In this paper, we propose a high-precision hybrid neural network model that employs Gated Recurrent Units (GRU) and Convolution Neural Network (CNN) to build a GRU-CNN model to forecast PV system output power. The proposed framework has two major phases. Firstly, the sample data is divided into training set and test set. For this, the temporal characteristics of the data set are extracted using a GRU model and the spatial characteristics are obtained using the CNN model. Secondly, the final predicted PV power is obtained through the output layer. The forecasting accuracy of GRU-CNN is determined by the mean absolute error (MAE), mean square error (MSE), determination coefficient $\left(\mathrm{R}^{2}\right)$ and root mean square error (RMSE) values. The findings of the comparison experiments show that the GRU-CNN model has better accuracy than some deep learning methods, including, GRU, CNN and long-short term memory model (LSTM).
\end{abstract}

\section{Introduction}

Recently, it is evident that the utilization of renewable energy sources has increased worldwide. This increase is due to the advantages of renewable energy sources and their impact on the environment, in addition to the huge increase in demand for load $[1,2]$. In particular, solar energy, as a source of renewable energy, is complete accessible, scalable and free [3]. Photovoltaic (PV) power generation can convert solar power into electric energy through the photovoltaic effect, which is one among the foremost promising renewable power generation techniques [4]. The photovoltaic energy plants have the major influence on the power increase in the world energy system. However, the random and intermittent nature of solar energy leads to difficulties in the stable operation and management of the power grid [5]. PV power forecasting is an important technology to consider in this instance, as it is able to minimize the gap between electricity supply and demand. At present, PV power prediction is one of the most economical and feasible solutions. Meanwhile, the accurate forecasting of $\mathrm{PV}$ power generation has positive effects on the control strategies of PV-battery energy storage systems, such as improving the self-consumption of PV systems and reducing power flows[6]. Many load forecasting approaches based on massive data have arisen as a result of this background, and these methods are primarily

\footnotetext{
*e-mail: sabri.mohamed195@gmail.com

**e-mail: mohamed.elhassouni@flsh.um5.ac.ma
}

grouped into three categories: 1) approaches of statistics; 2) physical methods; 3) artificial intelligence based methods [7]. Statistical methods use only historical PV power data as inputs, which are suitable for forecasting in the short and ultra-short term. forecasting, such instance time series method [8], autoregressive moving average (ARMA) and linear regression (LR) [9]. The physical method is a mathematical model based on the principle of photovoltaic power generation, [10]. The numerical weather prediction (NWP) information as inputs [11] or soft-computing models [12]. Analytical equations are usually complicated with high-computational cost [13]. Artificial intelligent learning methods, has the ability to benefit from the rapid growth of computing power and exploit artificial intelligence algorithm to map directly from input to output [14]. The primary methods for solar forecasting are persistence model, artificial neural network (ANN), support vector machine (SVM), and hybrid model [15].To estimate the power generation of PV generators almonacid et al. [16] proposed an ANN model (the multi-layer perceptron (MLP) network developed by the Hahn University Solar and Automated Energy team) and compared with classical methods. Cervone et al. [17] proposed a hybrid method based on neural network and analog ensemble for shortterm pv power forecasting for photovoltaic fields in three regions of Italy. Abdel-Nasser and Mahmoud [18] used a new deep long short term memory for forecasting the Photovoltaic power output. Takashi Kuremoto [19] used deep 
neural networks for time series forecasting. Gated recurrent unit (GRU) [20] is an advancement of the standard RNN based on optimized LSTM, and the internal unit of the GRU is identical to the LSTM[21]. In [22], a GRU model is utilized to investigate the temporal sequence of relationships in typhoon progression. Wang used novel approach based on Gated recurrent unit networks to forecast short-term Photovoltaic power [23]. Kim et al. proposed CNN-LSTM model to predict electricity and recorded the lowest error rates as compared to other classical models, because CNN-LSTM model learned from both spatial and temporal features [24]. However, although deep learning models are potential in the field of Photovoltaic power prediction, they still require to be further researched as there are limited existing studies currently concentrating on input feature construction and model training.

In this paper, a hybrid deep learning forecasting model is proposed for short term PV power forecasting with a five-minute interval. The proposed model is based on Gated Recurrent Units (GRU) and Convolution Neural Network (CNN). In the GRU-CNN, the GRU module is used to get the temporal features and CNN module is utilized to extract spatial features corresponding to its output GRU features. The main purpose of this work is to achieve more accurate PV power forecasting through the combination of GRU and CNN network. The validity of the GRU-CNN model proposed in this paper is assessed by comparison with GRU, LSTM, and CNN models. The proposed GRU-CNN model and the other models is assessed on mean absolute error (MAE), and mean square error (MSE), determination coefficient $\left(\mathrm{R}^{2}\right)$ and root mean square error (RMSE) values were used as the evaluation metrics.

This paper is organized as follows: in Section 2, briefly introduces and analyses the proposed GRU-CNN prediction model to analyse the temporal-spatial features. Section 3 shows the practical experimental results and comparisons for the evaluation of the of the GRU-CNN model. Finally, the conclusions and future work are summarized in Section 4.

\section{Method description}

\subsection{Gated Recurrent Unit}

The GRU is a type of recurrent neural network that is a newer generation and is a variant of LSTM. The GRU structure, as shown in Figure 1, by combining the forgetting gate and the input gate into an update gate, the LSTM three-gate design is improved, so there are one reset gate and two gates update gate in GRU which is aiming to optimize the cellular structure.

The composition and structure of cell structure effectively boosts the efficiency of the operation. So during training the GRU neural network converges faster than LSTM [25]. The calculation principle of each unit of GRU is as follows [26].A reset gate $r_{t}$ and an update gate $z_{t}$ are both included in the GRU, from the input of the current neuron $x_{t}$ to the update gate $z_{t}$, there is:

$$
z_{t}=\sigma\left(W_{x t} x_{t}+W_{h z} h_{t-1}+b_{z}\right)
$$

where $b_{z}$ is the bias of the update gate; $W_{x z}$ is the weight between the update gate and the input of the current neuron; $W_{h z}$ is the weight between the update gate and the hidden state $; h_{t-1}$ denotes the output of the previous neuron; $\sigma($.$) is the sigmoid function which can be determined$ as:

$$
\sigma=\frac{1}{1+e^{-x}}
$$

The reset gate $r_{t}$ is used to adjust the degree to which the previous moments status information is ignored.

$$
r_{t}=\sigma\left(W_{x r} x_{t}+W_{h r} h_{t-1}+b_{r}\right)
$$

where $b_{r}$ is the bias of the reset gate; $W_{x r}$ is the weight between the input of the current neuron and the reset gate; $W_{h r}$ is the weight between the hidden state and the reset gate.

The following formula can be used to calculate the candidate for the hidden state:

$$
\tilde{H}_{t}=\tanh \left[W_{x h} x_{t}+W\left(r_{t} * h_{t-1}\right)\right]
$$

where tanh is the hyperbolic tangent function that represents the element-wise multiplication; $W_{x h}$ is the weight between the input of the current neuron and the hidden state; $W$ is the temporary weight after the element-wise multiplication.

Furthermore, the hidden state of this neuron $h_{t}$ can be determined as:

$$
h_{t}=h_{t-1} *\left(1-z_{t}\right)+z_{t} * \tilde{H}_{t}
$$

After that,the final output state $y_{t}$ is calculated as:

$$
y_{t}=\sigma\left(W_{h o} h_{t}+b_{h}\right)
$$

where $b_{h}$ is the bias of the hidden state; $W_{h o}$ is the weight between the final output state and the hidden state.

\subsection{Convolutional Neural Network}

Convolutional Neural Network (CNN) is a class of deep neural networks that is mainly used such as video prediction, visual imagery, and time series classification [27] due to its excellent features extraction and reorganization. As proven by [28], the CNN model is a powerful deep learning technique which has the advantage to overcome the complex problems. Figure 2 shows the structure of CNN. The later usually consists of input layer, convolutional layers, pooling layers, fully-connected layers and output layer. The input layer feeds the data into the network. The data needs to be processed before to be transmitted to the input layer. The neurons of the input layer are usually relative to the dimension of input data. The convolutional layers apply a convolution operation to the input. It performs several convolution kernels to generate new feature maps, and also a bias is added. In most cases, features with a large dimension are produced following the 


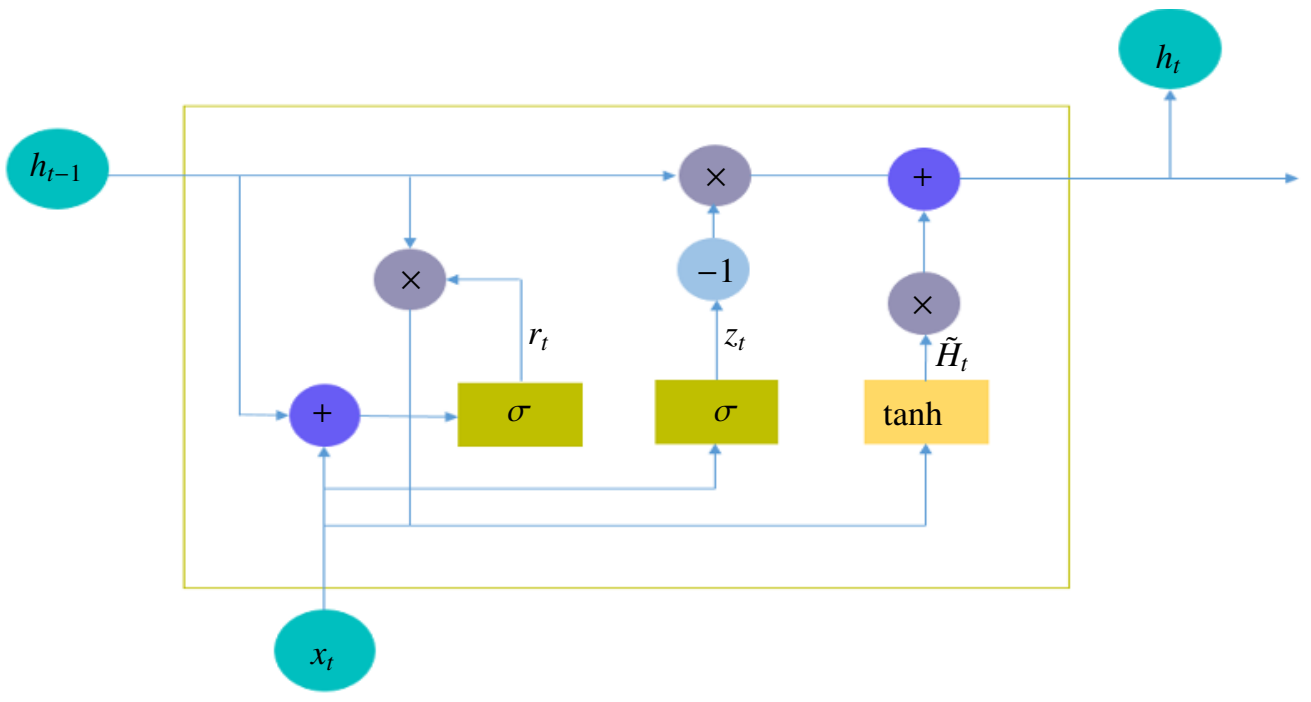

Figure 1: The GRU structure

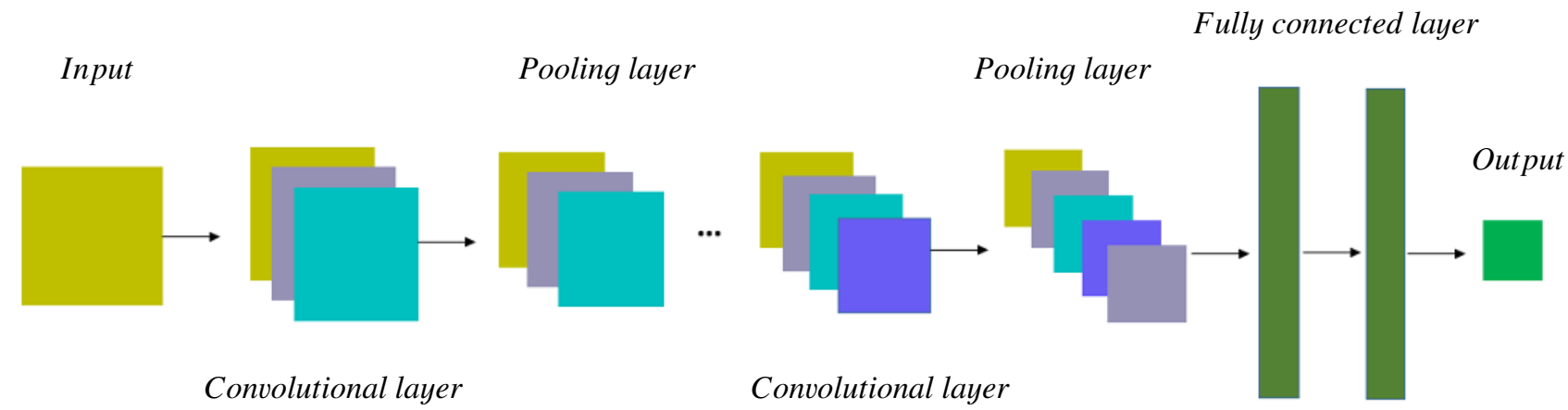

Figure 2: Convolutional Neural Networks (CNN) Architecture.

convolutional layer, which usually needs to reduce the inplane dimensionality of input maps. Thereby decreasing the number of learnable parameters and helping to avoid the overfitting issues. The pooling methods exist under different types, such as max pooling, and average pooling. Fully-connected layer is often used for high-level inference, which combines all local features into global features, and it is used to calculate the final result according to the following equation:

$$
y_{i}=\sum_{i=1}^{n} W_{i, j} x_{i}+b_{1}
$$

where $y_{j}$ is the output of fully-connected layer in the $j^{\text {th }}$ neuron, $n$ is the length of input data $(x), W_{i, j}$ stands for the neuron weight between $i_{\text {th }}$ input value and $j_{\text {th }}$ neuron and $b_{1}$ is the bias.

In this paper, the activation function selects the ReLU Rectified Linear Unit function which is defined as follows:

$$
k_{i}=g\left(y_{i}\right)=\max \left(0, y_{i}\right)
$$

where $k_{i}$ is the output after activation function.

\subsection{GRU-CNN Hybrid Neural Networks}

In this paper, a hybrid model called GRU-CNN is presented to predict PV power generation, which comprises of a serial connection of GRU and CNN, as shown in Fig.3. The first half of model GRU network, which is responsible to extracting the temporal characteristics of PV power, The second half is CNN network, which is responsible for extracting the characteristics spatial. The proposed model is highly capable of extracting complex features and patterns variables collected for PV power prediction. GRU processes the temporal feature in historical PV power data. GRU network main idea is to use specific neurons with a basic structure to store and transfer information over a long period of time in order to develop lasting memories, reduce the rate of information degradation, and detect long-term dependencies. The resulting temporal information collected by the GRU from the PV power time-series is then transmitted to the input layer of CNN model. The CNN convolutional layer performs a convolution operation on multivariate time-series data in order to extract spatial characteristics from various meteorological variables. A CNN often contains numerous levels of convolutional-pooling layers, with several convolution runs performed at each level to capture meaningful informa- 


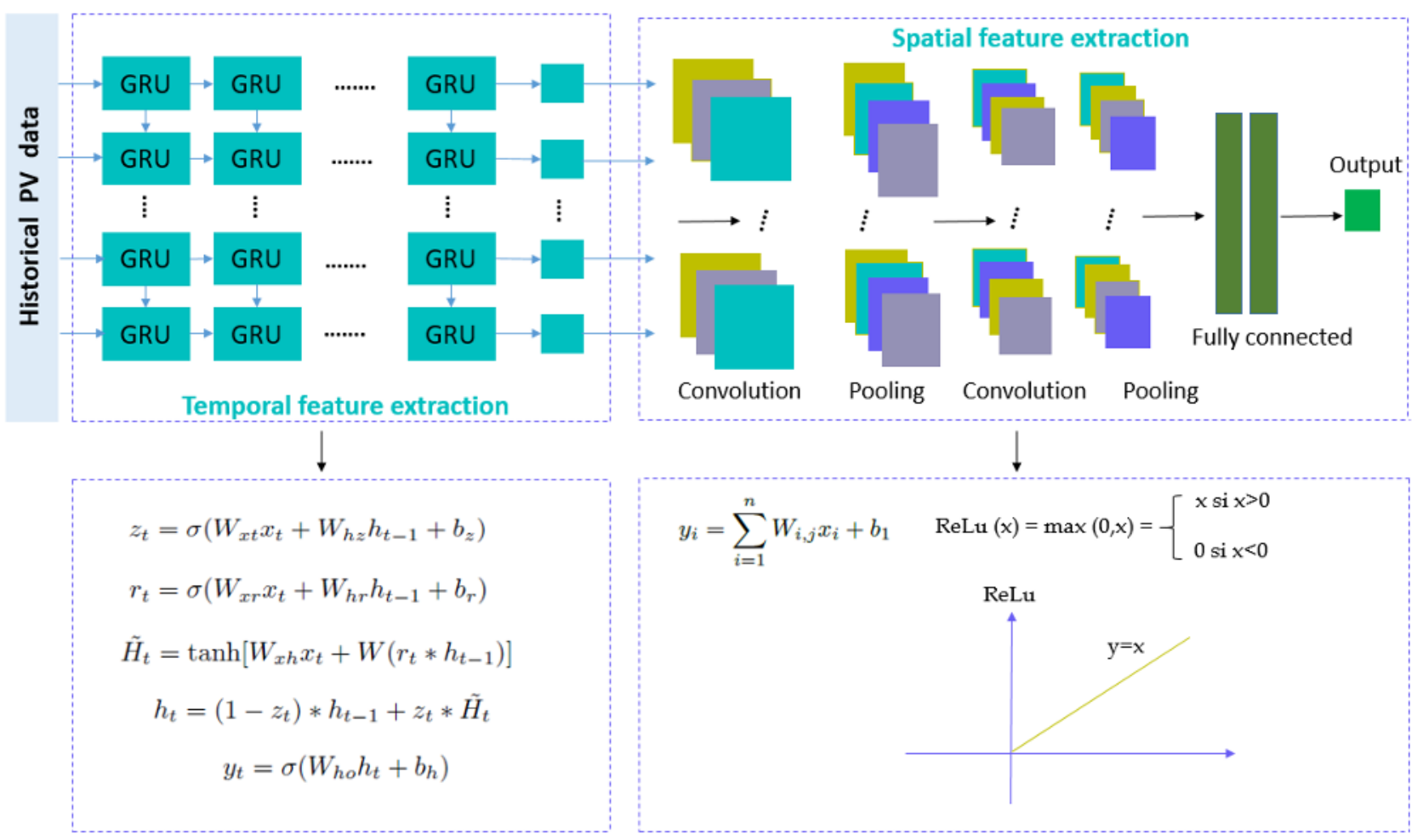

Figure 3: GRU-CNN hybrid neural networks.

tion. CNN applies weights to meteorological parameters based on their impact on PV power in this procedure. Finally, a fully connected (i.e., dense) layer is utilized to aggregate the data and forecast the PV power using extracted characteristics, as shown in Fig. 3

\subsection{Model Evaluation Indexes}

To compare the performance of different predictive models, we use the mean absolute Error (MAE), mean square error (MSE), and root mean square error (RMSE), and coefficient of determination $\left(\mathrm{R}^{2}\right)$. These evaluation criteria are defined as follows:

$$
\begin{gathered}
M A E=\frac{1}{N} \sum_{i=1}^{N}\left|y_{i}-\tilde{y}_{i}\right| \\
M S E=\frac{1}{N} \sum_{i=1}^{N}\left(y_{i}-\tilde{y}_{i}\right)^{2} \\
R M S E=\sqrt{\frac{1}{N} \sum_{i=1}^{n}\left(y_{i}-\tilde{y}_{i}\right)^{2}} \\
R^{2}=1-\frac{\sum_{i=1}^{N}\left(y_{i}-\tilde{y}_{i}\right)^{2}}{\sum_{i=1}^{N}\left(y_{i}-\bar{y}_{i}\right)^{2}}
\end{gathered}
$$

where $N$ is the total number of test samples. $y_{i}$ and $\tilde{y}_{i}$ are the measured and predicted output power, respectively. $\bar{y}_{i}$ is the average of the measured power in the test set. The predictive model is more efficient when $\mathrm{R}^{2}$ is closer to 1 . The lower are the values of MAE, MSE and RMSE, the greater is the prediction performance.
Table 1: Ranges of variables.

\begin{tabular}{ll}
\hline Variable & Ranges \\
\hline PV output power $(\mathrm{kW})$ & $0-19.35$ \\
Current Phase Average $(\mathrm{A})$ & $0-80.10$ \\
Global Horizontal Radiation $\left(\mathrm{W} / \mathrm{m}^{2}\right)$ & $0-2015.18$ \\
Diffuse Horizontal Radiation $\left(W / \mathrm{m}^{2}\right)$ & $0-769.85$ \\
Weather Temperature Celsius $\left({ }^{\circ} \mathrm{C}\right)$ & $-39.98-61.92$ \\
Wind Direction $\left(\hat{A}^{o}\right)$ & $-6.97-5140.88$ \\
Weather Relative Humidity $(\%)$ & $0-131.15$ \\
\hline
\end{tabular}

\section{Experiments and Results}

\subsection{The description of the experimental data}

In the simulation, the historical PV output power data are acquired from the Desert Knowledge Australia Solar Centre (DKASC). whose data can be openly accessed at [29]. The data used are from a manufacturer named $1 \mathrm{~B}$ DKASC, Alice Springs PV system. The dataset covers the period from May 2017 to May 2021, with a resolution of 5 minutes. The data includes current phase average (A), active power (KW), weather temperature celsius $\left({ }^{\circ} \mathrm{C}\right)$, diffuse horizontal radiation $\left(\mathrm{W} / \mathrm{m}^{2}\right)$, weather relative humidity $(\%)$, global horizontal radiation $\left(\mathrm{W} / \mathrm{m}^{2}\right)$, wind direction $\left(\hat{A}^{o}\right)$ etc, the ranges of the variables are shown in Table 1. The PV dataset contains a total of 414169 samples. In this study,the training set is made up of 331329 samples drawn from these databases. The test set also includes 82833 samples. The output PV power dataset is shown in figure 4. 


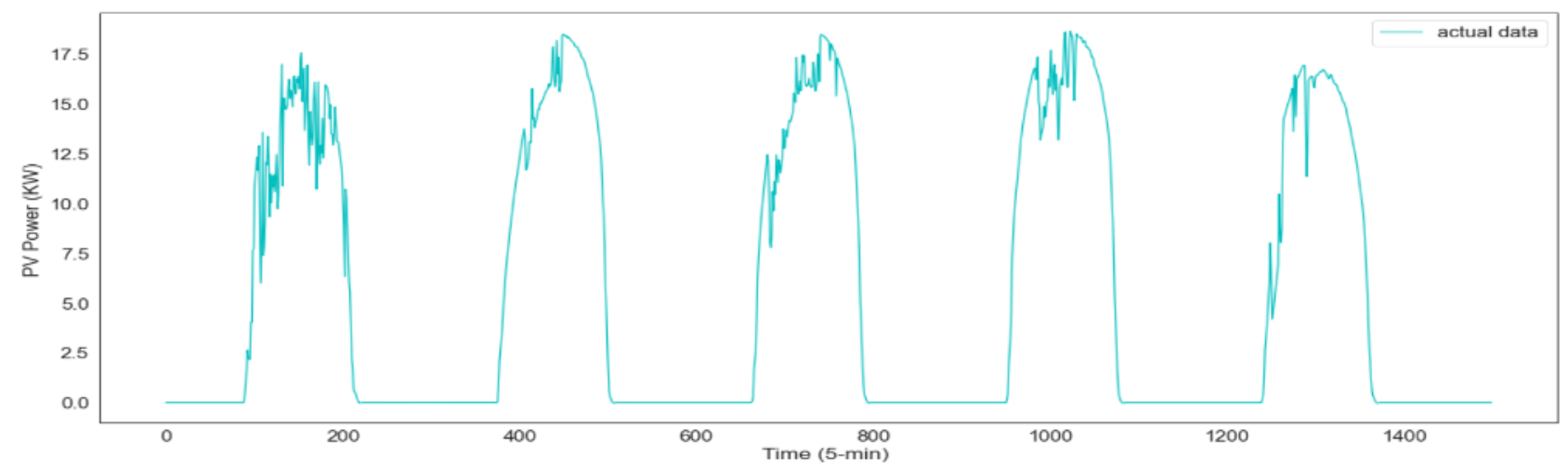

Figure 4: The output PV power in train dataset.

Table 2: The parameters settings in this paper.

\begin{tabular}{lll}
\hline Test & GRU & CNN \\
\hline Test-1 & units $=60$ & filters $=50 ;$ kernel-size $=2 ;$ stride $=2$ \\
Test- 2 & units $=80$ & filters $=100 ;$ kernel-size $=2 ;$ stride $=2$ \\
Test-3 & units $=80 ;$ Dropout $=0.2$ & filters $=100 ;$ kernel-size $=2 ;$ stride $=2 ;$ Dropout $=0.2$ \\
Test-4 & units $=80,100$ & filters $=200 ;$ kernel-size $=2 ;$ stride $=2$ \\
Test-5 & units $=100,150 ;$ Droupout $=0.2$ & filters $=32,64 ;$ kernel-size $=2 ;$ stride $=2 ;$ Dropout $=0.2$ \\
\hline & LSTM & GRU-CNN \\
\hline Test-1 & units $=60$ & units $=60 ;$ filters $=50 ;$ kernel-size $=2 ;$ stride $=2$ \\
Test-2 & units $=80$ & units $=80 ;$ filters $=100 ;$ kernel-size $=2 ;$ stride $=2$ \\
Test-3 & units $=80 ;$ Dropout $=0.2$ & units $=80 ;$ filters $=100 ;$ kernel-size $=2 ;$ stride $=2 ;$ Dropout $=0.2$ \\
Test-4 & units $=80,100$ & units $=80,100 ;$ filters $=200 ;$ kernel-size $=2 ;$ stride $=2$ \\
Test-5 & units $=100,150 ;$ Droupout $=0.2$ & units $=100,150 ;$ filters $=32,64 ;$ kernel-size $=2 ;$ stride $=2 ;$ Dropout $=0.2$ \\
\hline
\end{tabular}

\subsection{Simulation and results}

In this simulation, the performance of GRU-CNN forecasting model is evaluated comparing with three standard forecasting models : gated recurrent unit (GRU) model, convolutional neural network $(\mathrm{CNN})$ model, and longshort term memory model (LSTM) model. The parameters settings of the proposed network in this paper are shown in Table 2. For this experiment the value of batch size is 80 , number of iterations in each epoch is 100 . As shown in Tables 3-5, the CNN has the highest averaged MAE, MSE, and RMSE among the four models. The GRU module results are slightly better than the LSTM module, although they are both higher than the CNN. In more detail, CNN achieved values of $0.1486,0.0987$ and 0.2912 for the averaged MAE, MSE and RMSE, respectively, and GRU achieved values of $0.1116,0.0319$ and 0.1766 for the averaged MAE, MSE and RMSE, while LSTM reduced its error rate to $0.1039,0.0358$ and 0.1861 for the averaged MAE, MSE and RMSE. The suggested GRU-CNN model, which combines the GRU and CNN models, produces the most accurate forecasts. The proposed GRU-CNN models average indexes are the lowest among all models: 0.0813, 0.0194 and 0.1359 . However, the obtained results verified the practicality of the proposed model in the photovoltaic system power forecasting.

In practice, accurate forecasting of photovoltaic power helps to reduce the uncertainty and volatility in the estima-
Table 3: MAE of different models.

\begin{tabular}{lllll}
\hline Test & GRU & LSTM & CNN & GRU-CNN \\
\hline Test-1 & 0.1052 & 0.1033 & 0.1047 & 0.0878 \\
Test-2 & 0.0886 & 0.1196 & 0.1967 & 0.0852 \\
Test-3 & 0.1635 & 0.1050 & 0.1260 & 0.0709 \\
Test-4 & 0.0829 & 0.0958 & 0.1193 & 0.0755 \\
Test-5 & 0.1125 & 0.0959 & 0.1964 & 0.0872 \\
Test-Avg & 0.1116 & 0.1039 & 0.1486 & 0.0813 \\
\hline
\end{tabular}

Table 4: MSE of different models.

\begin{tabular}{lllll}
\hline Test & GRU & LSTM & CNN & GRU-CNN \\
\hline Test-1 & 0.0366 & 0.0382 & 0.0487 & 0.0305 \\
Test-2 & 0.0226 & 0.0536 & 0.0763 & 0.0236 \\
Test-3 & 0.0492 & 0.0245 & 0.0459 & 0.0118 \\
Test-4 & 0.0220 & 0.0446 & 0.0493 & 0.0194 \\
Test-5 & 0.0291 & 0.0183 & 0.2735 & 0.0174 \\
Test-Avg & 0.0319 & 0.0358 & 0.0987 & 0.0194 \\
\hline
\end{tabular}

tion of photovoltaic power. To verify the competitiveness of the proposed model further, $\mathrm{R}^{2}$ is used to measure the performance in the four models. Note that the predictive model will be more effective when $\mathrm{R}^{2}$ closer to 1 . Table 6 shows the $\mathrm{R}^{2}$ for the four model, The GRU-CNN model 

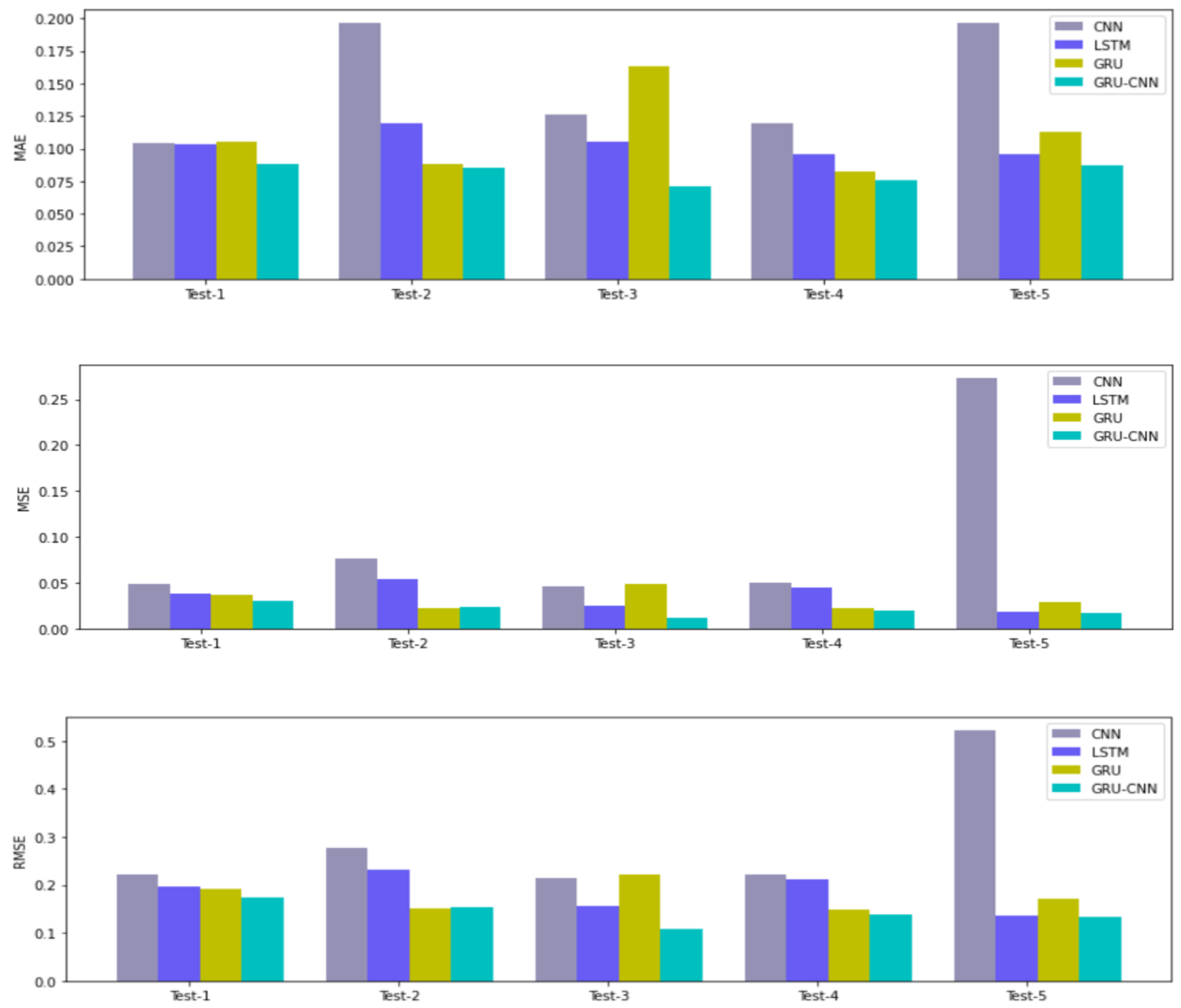

Figure 5: Performance evaluations of different models for each test

Table 5: RMSE of different models.

\begin{tabular}{lllll}
\hline Test & GRU & LSTM & CNN & GRU-CNN \\
\hline Test-1 & 0.1914 & 0.1954 & 0.2207 & 0.1748 \\
Test-2 & 0.1505 & 0.2316 & 0.2763 & 0.1544 \\
Test-3 & 0.2219 & 0.1567 & 0.2143 & 0.1088 \\
Test-4 & 0.1484 & 0.2113 & 0.2222 & 0.1394 \\
Test-5 & 0.1708 & 0.1355 & 0.5229 & 0.1322 \\
Test-Avg & 0.1766 & 0.1861 & 0.2912 & 0.1359 \\
\hline
\end{tabular}

Table 6: $R^{2}$ of different models.

\begin{tabular}{lllll}
\hline Test & GRU & LSTM & CNN & GRU-CNN \\
\hline Test-1 & 0.9981 & 0.9980 & 0.9975 & 0.9984 \\
Test-2 & 0.9988 & 0.9973 & 0.9961 & 0.9988 \\
Test-3 & 0.9975 & 0.9987 & 0.9976 & 0.9994 \\
Test-4 & 0.9988 & 0.9977 & 0.9975 & 0.9990 \\
Test-5 & 0.9985 & 0.9990 & 0.9862 & 0.9991 \\
Test-Avg & 0.9983 & 0.9981 & 0.9949 & 0.9989 \\
\hline
\end{tabular}

average $\mathrm{R}^{2}$ value is 0.9989 , which is higher than the GRU model $\mathrm{R}^{2}$ is 0.9983 , which is better than the LSTM model. $\mathrm{R}^{2}$ is 0.9981 , which is better than the CNN model $\mathrm{R}^{2}$ is 0.9983 . According to the average indexes, as shown by the average indexes, Our suggested GRU-CNN model can reach the best performance in PV Forecasting. For a better visualization, the results of the models are also illustrated in Figure 5.

The proposed GRU-CNN model has the smallest bar of all the models. In test 1 , test 3 , and test 5, the MAE, MSE, and RMSE bar of LSTM outperform the GRU model. Although the GRU and LSTM model performs always better than the CNN model but is inferior to GRUCNN model.It is clear that the suggested model performs well in all five partitions, demonstrating that the proposed GRU-CNN model can increase PV power forecast stability over the other models.

As can be seen from Tables 3-6, among all the models, the input sequence of test 3 on the testing set shows the best effect. In order to better show the predicting results of the proposed model in this paper, Figure 6 reports the 


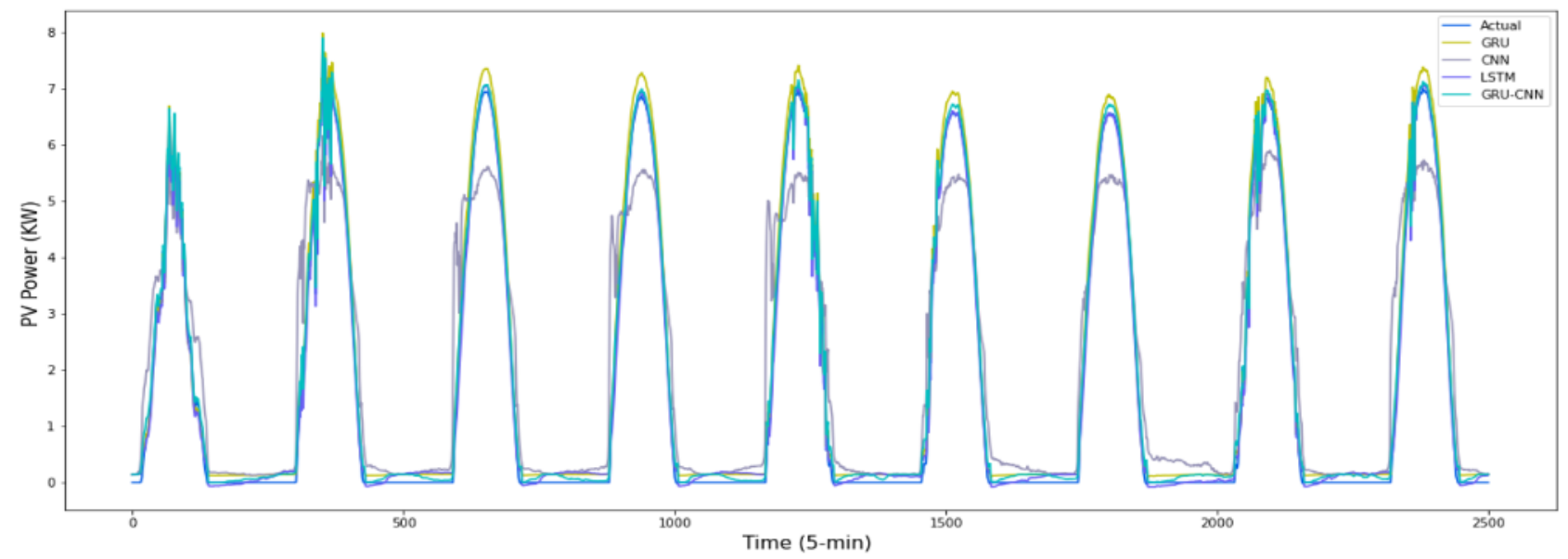

Figure 6: Forecasting the PV power using all the methods in the input sequence of test 3.

forecasting results of the four models and actual PV power in the input sequence of test 3 . The predicting results of all of the models outputs follow the same pattern as the real values. The proposed GRU-CNN model has a higher similarity, forecasting results is the closest to the actual value.

In this paper, we proposed a new hybrid deep neural network framework that combines the GRU and CNN modules. In the experiments, GRU provide more accurate results than LSTM and CNN but our proposed GRU-CNN model achieves the smallest error rate when compared to other baseline models. Furthermore, the dataset is separated into train and test data with different input sequence to express the performance of the proposed model more intuitively. All of the tests show that the proposed model performs well, it shows that combining the gated recurrent unit (GRU) with convolutional neural networks (CNN) can improve prediction stability and effectiveness in $\mathrm{PV}$ power forecasting, this work proposes a new research approach in time sequence forecasting. Future research could aim to enhance the accuracy of short-term PV power forecasts by incorporating the hidden features of GRU and CNN in a more effective way.

\section{Conclusion}

This paper proposes a new short term prediction model based on GRU and CNN, which mainly introduces the application of GRU-CNN in short-photovoltaic power prediction. The proposed model is tested on 1B DKASC, Alice Springs PV system data which are publicly available. Due to the non-linearity in the input data, we transform it by applying a standard minmax scalar then fed the standardized data for further training processes. After, we developed a hybrid model by employing GRU for temporal features representation and $\mathrm{CNN}$ for spatial features representation to achieve the lowest error rates when compared to the traditional methods. Different evaluation matrices were used to validate the stability, accuracy, and performance of the proposed GRU-CNN model. The experimental results show that the proposed GRU-CNN based pre- diction model is superior to other baseline models such as GRU, LSTM and CNN. It has higher prediction accuracy. The future work of this study will includes further improving the accuracy and stability of our proposed method and applying it on many datasets.

\section{References}

[1] Ellabban, O., Abu-Rub, H. \& Blaabjerg, F. Renewable energy resources: Current status, future prospects and their enabling technology. Renewable and sustainable energy reviews 39, 748-764 (2014).

[2] Mahmud, N. \& Zahedi, A. Review of control strategies for voltage regulation of the smart distribution network with high penetration of renewable distributed generation. Renewable and Sustainable Energy Reviews 64, 582-595 (2016).

[3] Sahu, B. K. A study on global solar PV energy developments and policies with special focus on the top ten solar PV power producing countries. Renewable and Sustainable Energy Reviews 43, 621-634 (2015).

[4] Wang, F. et al. Image phase shift invariance based cloud motion displacement vector calculation method for ultra-short-term solar PV power forecasting. Energy conversion and management 157, 123-135 (2018).

[5] Li, G., Wang, H., Zhang, S., Xin, J. \& Liu, H. Recurrent neural networks based photovoltaic power forecasting approach. Energies 12, 2538 (2019).

[6] Litjens, G., Worrell, E. \& van Sark, W. Assessment of forecasting methods on performance of photovoltaic-battery systems. Applied Energy 221, 358- 373 (2018).

[7] Wang, K., Qi, X. \& Liu, H. A comparison of dayahead photovoltaic power forecasting models based on deep learning neural network. Applied Energy 251, 113315 (2019).

[8] Box, G. E., Jenkins, G. M., Reinsel, G. C. \& Ljung, G. M.Time series analysis: forecasting and control (John Wiley \& Sons, 2015). 
[9] Nury, A. H., Hasan, K. \& Alam, M. J. B. Comparative study of wavelet- ARIMA and wavelet-ANN models for temperature time series data in northeastern Bangladesh. Journal of King Saud UniversityScience 29, 47-61 (2017).

[10] Inman, R. H., Pedro, H. T. \& Coimbra, C. F. Solar forecasting methods for renewable energy integration. Progress in energy and combustion science 39, 535-576 (2013).

[11] Yang, D., Kleissl, J., Gueymard, C. A., Pedro, H. T. \& Coimbra, C. F. History and trends in solar irradiance and PV power forecasting: A preliminary assessment and review using text mining. Solar Energy 168, 60- 101 (2018).

[12] Rafique, S. F. \& Jianhua, Z. Energy management system, generation and demand predictors: a review. IET Generation, Transmission \& Distribution 12, 519-530 (2018).

[13] Alonso-Montesinos, J. \& Batlles, F. J. Solar radiation forecasting in the short term under all sky conditions. Solar Energy Energy 83, 387-393 (2015).

[14] Hossain, M., Mekhilef, S., Danesh, M., Olatomiwa, L. \& Shamshirband, S. Application of extreme learning machine for short term output power forecasting of three grid-connected PV systems. Solar Energyjournal of Cleaner Production 167, 395-405 (2017).

[15] Das, U. K. et al. Forecasting of photovoltaic power generation and model optimization: A review. Renewable and Sustainable Energy Reviews 81, 912-928 (2018).

[16] Almonacid, F., Rus, C., P'erez-Higueras, P. \& Hontoria, L. Calculation of the energy provided by a PV generator. Comparative study: Conventional methods vs. artificial neural networks. Energy 36, 375-384 (2011).

[17] Cervone, G., Clemente-Harding, L., Alessandrini, S. \& Delle Monache, L. Short-term photovoltaic power forecasting using Artificial Neural Networks and an Analog Ensemble. Renewable Energy 108, 274-286 (2017).

[18] Abdel-Nasser, M. \& Mahmoud, K. Accurate photovoltaic power forecasting models using deep LSTM-RNN. Neural Computing and Applications
31,2727-2740 (2019).

[19] Kuremoto, T., Kimura, S., Kobayashi, K. \& Obayashi, M. Time series forecasting using a deep belief network with restricted Boltzmann machines. Neurocomputing 137, 47-56 (2014).

[20] Chung, J., Gulcehre, C., Cho, K. \& Bengio, Y. Empirical evaluation of gated recurrent neural networks on sequence modeling. arXiv preprint arXiv:1412.3555 (2014).

[21] Cheng, H., Xie, Z., Wu, L., Yu, Z. \& Li, R. Data prediction model in wireless sensor networks based on bidirectional LSTM. EURASIP Journal on Wireless Communications and Networking 2019, 1-12 (2019).

[22] Dong, P., Lian, J. \& Zhang, Y. A novel datadriven approach for tropical cyclone tracks prediction based on Granger causality and GRU in 2019 IEEE International Conference on Service Operations and Logistics, and Informatics (SOLI) (2019), 70-75.

[23] Wang, Y., Liao, W. \& Chang, Y. Gated recurrent unit network-based shortterm photovoltaic forecasting. Energies 11, 2163 (2018).

[24] Kim, T.-Y. \& Cho, S.-B. Predicting residential energy consumption using CNN-LSTM neural networks. Energy 182, 72-81 (2019).

[25] Gao, X. et al. Short-term electricity load forecasting model based on EMDGRU with feature selection. Energies 12 , 1140 (2019).

[26] Cheng, L. et al. Ensemble recurrent neural network based probabilistic wind speed forecasting approach. Energies 11 , 1958 (2018).

[27] Shao, X., Kim, C. S. \& Kim, D. G. Accurate multiscale feature fusion $\mathrm{CNN}$ for time series classification in smart factory. Comput., Mater. Continua 65, 543-561 (2020).

[28] He, K., Zhang, X., Ren, S. \& Sun, J. Delving deep into rectifiers: Surpassing human-level performance on imagenet classification in Proceedings of the IEEE international conference on computer vision (2015), 1026-1034.

[29] http://dkasolarcentre.com.au/locations/alicesprings? source $=1 \mathrm{~B}$. 